\title{
Hidden Terminal-Aware Contention Resolution with an Optimal Distribution
}

\author{
Euhanna Ghadimi, Pablo Soldati, Fredrik Österlind, Haibo Zhang and Mikael Johansson
}

\begin{abstract}
Achieving low-power operation in wireless sensor networks with high data load or bursty traffic is challenging. The hidden terminal problem is aggravated with increased amounts of data in which traditional backoff-based contention resolution mechanisms fail or induce high latency and energy costs. We analyze and optimize Strawman, a receiver-initiated contention resolution mechanism that copes with hidden terminals. We propose new techniques to boost the performance of Strawman while keeping the resolution overhead small. We finally validate our improved mechanism via experiments.
\end{abstract}

\section{INTRODUCTION}

Wireless sensor networks experience traffic bursts due to route [1] and code [2] updates, bulk transfers [3], and spatiallytemporally correlated events [4]. Traffic bursts aggravate the hidden terminal problem, as nodes that are hidden to each other may attempt to simultaneously send data to the same neighbor, causing data collisions and losses. The emerging class of receiver-initiated duty-cycled MAC protocols [5], [6], [7] promises both reduced congestion and improved resilience against hidden terminals, in comparison to traditional senderinitiated protocols [8], [6]. In particular, the Strawman [7] contention resolution mechanism - designed for receiverinitiated duty-cycled protocols - mitigates the hidden terminal problem through an RTS/CTS-like handshake.

With the recently proposed Strawman contention resolution protocol [7] as outset, we analyze its key component for efficiently coping with hidden terminals: the distribution used to generate random-length packets. We propose improvements to Strawman that increase both throughput and scalability. We demonstrate improved performance with extensive simulations, and validate our models on real hardware.

The contention resolution mechanism is at the core of dutycycled low-power wireless protocols, where it is responsible for resolving data packet collisions. Traditional contention resolution mechanisms are backoff-based and are suspectible to hidden terminals. Request-To-Send/Clear-To-Send mechanisms (RTS/CTS) have long been employed to mitigate the hidden terminal problem, but they suffer from high overhead in low-power sensor networks [9].

Strawman solves the hidden terminal problem efficiently by measuring which of multiple colliding random-length RTS transmissions is the longest. The contender that (randomly)

E. Ghadimi, P. Soldati, H. Zhang and M. Johansson are with the School of Electrical Engineering, Royal Institute of Technology, KTH, Stockholm, Sweden. $\{$ euhanna, soldati, haibo, mikaelj\}dee.kth.se

F. Österlind is with Swedish Institute of Computer Science (SICS), Stockholm, Sweden. frosasics. se picks the longest length is granted channel access and sends its data.

While Strawman has many promising properties, the initial design also has some drawbacks that limits its throughput and scalability. One such drawback is the use of a uniform distribution to draw request length. In this paper we improve Strawman by deriving the optimal request length distribution.

This paper contains three main contributions. First, we model the basic Strawman mechanism and derive an enhanced version, still based on the uniform distribution. Second, we design, analyze and evaluate an optimal non-uniform requestlength distribution which outperforms the uniform distribution. We also derive an approximation for Strawman that better suits sensor networks. Third, through extensive simulations, we demonstrate how hidden terminals and the capture-effect affects our contention-resolution mechanisms.

This paper is structured as follows. After reviewing contention resolution for sensor networks in Section II, we quantify the amount of hidden terminals and their impact in a sensor network testbed in Section III. We define a novel hidden terminal metric, and run a set of experiments on the publicly available TWIST sensor network testbed [11] to extract its hidden terminal profile. With our TWIST-profile, we show that contention resolution in sensor networks must handle the hidden terminal problem, or risk significant performance penalties. Section IV models the basic Strawman protocol, which we then improve in Section V. Section VI evaluates and compares our improved Strawman mechanisms.

\section{Contention Resolution}

Contention resolution in low-power wireless networks must both have low overhead and cope with hidden terminals. This section gives an overview of state-of-the-art contention resolution in sensor networks.

\section{A. Sender-initiated vs Receiver-initiated protocols}

Contention-based medium access protocols (MACs) can be partitioned into two classes: sender-initiated and receiverinitiated. In sender-initiated protocols, the sender initiates a new data transfer by a radio transmission. For example, Carrier Sense Multiple Access (CSMA) protocols belong to the sender-initiated class. Low-power sender-initiated protocols typically employ Low-Power Listening (LPL) [9]. LPL protocols use packet trains to implement a prolonged preamble, that the intended receiver can detect while duty-cycling the radio hardware. LPL has successfully been implemented on packet-based IEEE 802.15.4 CC2420 radio [12], [8]. 
In receiver-initiated protocols [5], [13], [6], [14] the receiver initiates a new data transfer by transmitting a data probe packet to all neighboring nodes. The probe is sometimes referred to as a Ready-To-Receive (RTR) packet. Nodes with receiver-destined data immediately transmit their data upon receiving a probe. If receiver-side collisions are detected, the receiver includes a random backoff window in the probe that coupled with sender-side physical carrier sensing implements a contention resolution mechanism similar to CSMA. The lowpower technique used in receiver-initiated protocols is called Low Power Probing (LPP). Receiver-initiated protocols, in comparison to sender-initiated, thus induces a fixed (but small) overhead by the periodic probe transmissions, but avoids the excessive packet transmissions associated with LPL's packet trains. Moreover, receiver-initiated protocols offer lower congestion and higher throughput in certain scenarios [13].

\section{B. The Hidden Terminal Problem}

The hidden terminal problem [15] arises in wireless networks where the individual node radio ranges do not cover the full network, such as in multi-hop sensor networks. More specifically, hidden terminals are nodes that are outside radio range of each other, but can communicate with a common "middle" node e.g., a base station in 802.11. Contention resolution based on physical carrier sensing (e.g. CSMA) that does not handle hidden terminals risk packet collisions.

Request-To-Send/Clear-To-Send (RTS/CTS) is a mechanism derived specifically to solve the hidden terminal problem. The sender, prior to transmitting its data packet, first requests channel access by an RTS transmission. The receiver replies with a CTS transmission (if the channel is available). All neighboring nodes refrain from transmissions throughout the data transfer. RTS/CTS mechanisms have been implemented in sensor networks [16], but has been shown to induce a high overhead due to the small data payloads used in sensor networks [9]. Moreover, although the RTS/CTS mechanism can solve the hidden terminal problem, it relies on all neighbors to be able to overhear the RTS/CTS control packets, but overhearing is not supported in modern duty-cycled networks [17].

The hidden terminal problem is aggravated in sensor networks with bursty traffic patterns. If the traffic is sparse, the occurrence of the hidden terminals may be lessened by adjusting the duty-cycle configuration [18], [8]. However, in networks with bursty traffic, such as alarm networks that have both spatially and temporally correlated traffic [19], a node must be able to efficiently receive large amounts of data from neighbors that may be hidden to each other.

Increasing the physical carrier sensitivity has been proposed to reduce the amount of hidden terminals [20], as senders get more sensitive to hearing each others transmissions. This approach may indeed remove hidden terminals, but also reduces the overall network capacity when applied to sensor networks. Since sensor networks typically span a much larger area than is covered by a single node, the exposed terminal problem is aggravated as the carrier sensitivity is increased: weak ongoing transmissions hinder new transmissions. Finally,

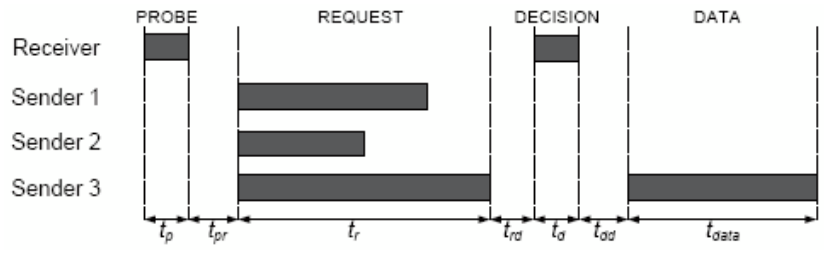

Fig. 1. The Strawman contention resolution mechanism grants channel access to the contender with the longest request transmissions.

hidden terminals may be very difficult to remove by increased carrier sensitivity due to asymmetric links [21].

\section{Capture effect}

The discussion has hitherto considered packet collisions as lost or corrupted data; if two nodes' transmissions overlap in time the receiver will not correctly receive any of them. The capture effect phenomenon allows a radio to correctly receive a data transmission even with simultaneous colliding transmissions. The capture effect requires that (1) the overlapping transmissions differ in signal strength, and (2) that the stronger transmission is initiated before the interfering weaker transmission(s). Dutta et al. exploit the capture effect to implement the network primitive backcast [6], and show that the capture effect is effective on the CC2420 radio chip as long as the signal strength difference is above $3 \mathrm{~dB}$.

\section{Strawman}

The Strawman protocol [7] is illustrated in Figure 1. Each Strawman contention period consists of four consecutive messages: PROBE, REQUEST, DECISION, and DATA. The receiver broadcasts a Strawman PROBE message to notify neighbors that it is ready to receive data. All neighbors that have data for the receiver contend for the channel by sending an immediate REQUEST. Multiple REQUESTs may thus collide at the receiver. The length of each REQUEST message is chosen randomly by sampling from a uniform distribution[7]. The receiver samples the channel for activity during the REQUESTS, and estimates the payload length of the longest REQUEST. The receiver then sends a DECISION message containing the length estimate. The contender whose REQUEST length matches the one specified in the DECISION is granted channel access, and sends its DATA message. Another contention round is initiated when the DATA has been received, or after a timeout. The PROBE message has dual purpose: it also acknowledges the last received DATA packet. Note that if two contenders pick the same random length, and hence are both granted channel access leading to a DATA collision, the timeout will trigger another contention period, and both data packets will be retransmitted due to the lack of acknowledgement. Note also that the Strawman contention resolution is used only if a receiver detects a data collision, and thus otherwise has zero overhead. Strawman has experimentally been shown to have high performance, and to mitigate the hidden terminal problem [7]. In the following sections we model and analyze the Strawman mechanism. 


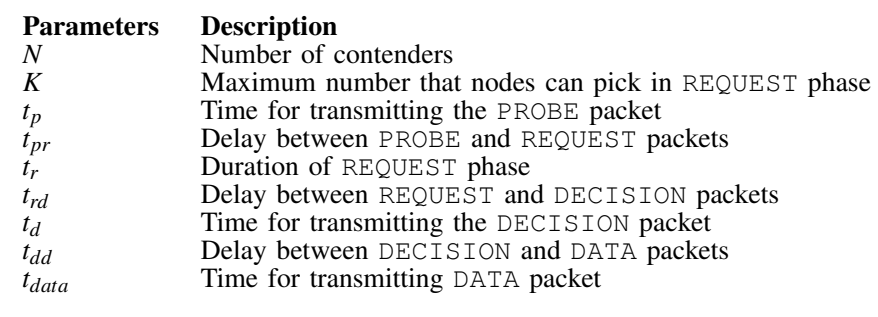

From our analysis, we improve Strawman with an optimal nonuniform request length distribution that significantly improves system goodput as well as scalability.

\section{A Study On Hidden Terminals}

Contention resolution mechanisms unable to handle the hidden terminal problem risk severe performance degradation in networks where hidden terminals do exist. This section demonstrates a novel hidden terminal metric. We will again use this metric to also evaluate Strawman in Section VI.

\section{A. The Hidden Terminal Metric}

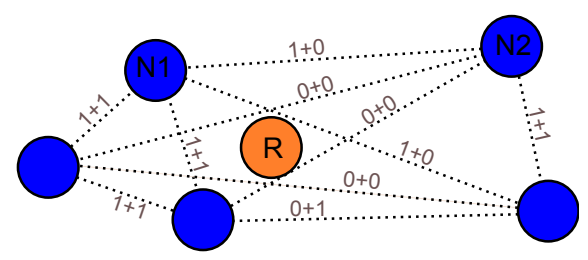

Fig. 2. The receiver-specific hidden terminal metric is based on the receiver's (R) neighbors' interconnections. The metric is defined as the complement of the number of (detectable) neighbor links, divided by all possible links. R's hidden terminal metric shown in the figure is: $1-11 /(5 \cdot 4)=45 \%$.

We define a hidden terminal metric to allow controlled evaluation of the impact of hidden terminals on contention resolution mechanisms. The metric represents how well the neighbors can detect each other's transmissions, see Figure 2. The metric is receiver-specific: different network nodes may be subject to different amounts of hidden terminals.

Given a receiver $R$, we define R's neighbors as the set of nodes that have a Packet Reception Ratio (PRR) to R above a fixed theshold. In Figure 2, neighbor N1 can detect N2's transmissions $(+1)$, but $\mathrm{N} 2$ can not detect N1's transmissions $(+0)$. The total number of detectable links in the example is 11 , and so the resulting hidden terminal metric is $45 \%$ (If all neighbors could detect all others' transmissions, the hidden terminal metric would be $0 \%$ ). Note that a detectable link may have a very low PRR, as long as the transmission can be detected using physical carrier sensing.

\section{B. Profiling a Testbed for Existence of Hidden Terminals}

We extract a realistic range of hidden terminal metrics by performing a set of experiments on the publicly available TWIST sensor network testbed [11]. TWIST has 102 CC2420equipped sensor nodes. We define the minimum link layer PRR theshold as 1/16 - a commonly used threshold in collection protocols. We use the default physical carrier sense threshold on the CC2420: $-77 \mathrm{dBm}$.
Our experiments on TWIST show that the hidden terminal metric varies significantly among different nodes in the network. The hidden terminal metrics in the TWIST testbed ranges between $11.0 \%$ and $29.4 \%$. Using our experiment traces, we furthermore model the capture effect effectiveness by the amount of links that differ with more than $3 \mathrm{~dB}$.

\section{Hidden terminals on naive random backoff}

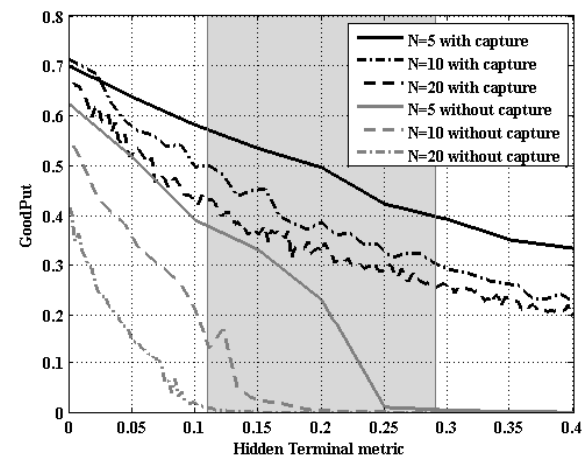

Fig. 3. The performance of CSMA with random backoff degrades significantly with increased hidden terminals. The figure shows the range of hidden terminals found in the TWIST testbed experiments.

Using our hidden terminal metric, we now quantify the impact of hidden terminals on a simulated CSMA-based star topology with a single receiver, see Figure 3 . The performance degradation is clearly visible with increasing hidden terminals, both with and without simulated capture effect. Note that although the addition of capture effect improves performance, the CSMA mechanism now fails to achieve one of its main objectives: fair contention resolution among contenders. Rather, when relying on capture effect, only the strongest of the contenders will transfer uncorrupted data.

\section{STRaWman Model}

In what follows, we model and analyze the Strawman mechanism [7], and we propose simple and effective modifications to improve its performance. Even though the conventional load of the WSN is meant to be low to moderate, Strawman is triggered to cope with sudden surges of the traffic.

In this regard we consider a snapshot of the network with a receiver node and a set of transmitters, labelled $n=1, \ldots, N$, that contend to access the channel. Upon detecting a collision, the receiver sends a Strawman PROBE packet as illustrated in Figure 1. Transmitters now contend for the channel by sending a REQUEST packet with random length $x_{n}$ chosen with uniform distribution $x_{n} \sim \mathscr{U}[1, K]$, where the maximum length $K$ is referred to as the Strawman resolution.

Given the number of contenders $N$ and the resolution $K$, the success probability $P_{N, K}$ of a Strawman round is the probability that one contender draws a number $x_{n}=k$ with $k \in[1, K]$ while all other contenders draw smaller numbers, i.e.

$$
\begin{aligned}
P_{N, K} & =\sum_{k=1}^{K} \sum_{n=1}^{N} \operatorname{Prob}\left\{x_{n}=k, x_{j}<k \forall j \neq n\right\} \\
& =\frac{N}{K^{N}} \sum_{k=1}^{K}(k-1)^{N-1} .
\end{aligned}
$$




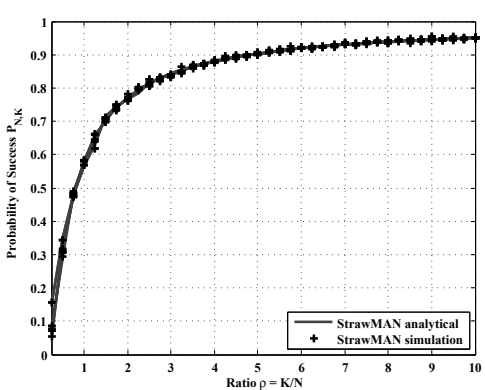

(a) Round success probability

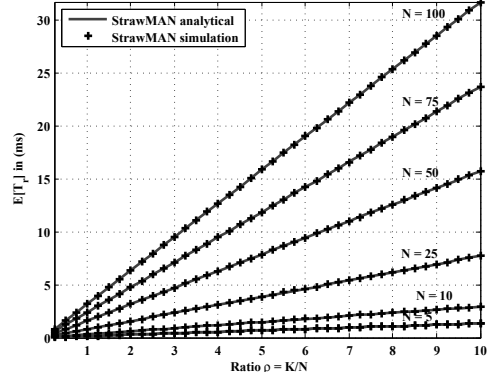

(b) Average request length.

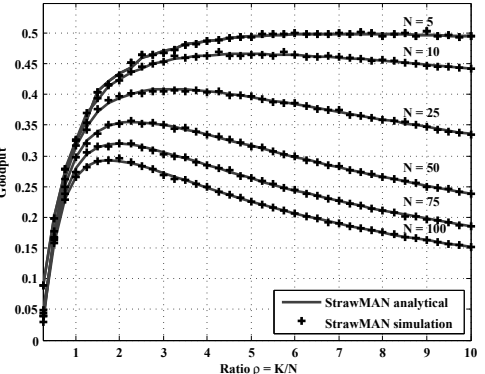

(c) Goodput.

Fig. 4. Validation of the analytical model for Strawman contention resolution with uniform distribution. We plot the success probability $P_{N, K}$ of a Strawman round, the average request length $E\left[t_{r}\right]$ and the goodput $g$ against the ratio $\rho=K / N$ for various number of contenders $N=\{5,10,25,50,75,100\}$.

The probability of successfully receiving a packet over $m$ consecutive Strawman rounds follows a geometric distribution as

$$
P_{S}(m)=1-\left(1-P_{N, K}\right)^{m} \text {. }
$$

Let $x \in[1, K]$ be the length of the largest REQUEST packet. Then, the average length of a request round is

$$
\begin{aligned}
E[x] & =\sum_{k=1}^{K} k \cdot \operatorname{Prob}\{x=k\} \\
& =\sum_{k=1}^{K} k \cdot \operatorname{Prob}\{x \leq k\} \cdot(1-\operatorname{Prob}\{x<k \mid x \leq k\}) \\
& =\sum_{k=1}^{K} k\left(\frac{k}{K}\right)^{N}\left[1-\left(\frac{k-1}{k}\right)^{N}\right] .
\end{aligned}
$$

where $\operatorname{Prob}\{x<k \mid x \leq k\}$ is the probability that the largest number among all contenders is smaller than $k$ conditioned to the event that $k$ is the maximum number that can be drawn. Then, the average time duration $E\left[t_{r}\right]$ of a REQUEST phase is

$$
E\left[t_{r}\right]=t_{b} E[x],
$$

where $t_{b}$ is the time to transmit one byte of data; for IEEE 802.15.4-compatible radios that transmit at $250 \mathrm{kbps}, t_{b}=\frac{8}{250}$. Similarly, let $\tilde{N}$ be the number of contenders winning a Strawman round, then

$$
\begin{aligned}
E[\tilde{N}] & =\sum_{n=1}^{N} n \cdot \operatorname{Prob}\left\{\exists n \text { nodes with } x_{n}=k, x_{j}<k \text { otherwise }\right\} \\
& =\sum_{n=1}^{N} n\left(\begin{array}{c}
N \\
n
\end{array}\right) \sum_{k=1}^{K}\left(\frac{1}{K}\right)^{n}\left(\frac{k-1}{K}\right)^{N-n} .
\end{aligned}
$$

Let $t_{0} \triangleq t_{p}+t_{p r}+t_{r d}+t_{d}+t_{d d}$ denote the constant part of the access delay in a Strawman round (all phases apart from the REQUEST phase). Then, the expected round length is

$$
E\left[T_{\text {round }}\right]=E\left[t_{r}\right]+t_{\text {data }}+t_{0} .
$$

Finally, we define the goodput as the portion of network-layer transmitted traffic per round:

$$
\begin{aligned}
g & =\frac{P_{N, K}}{E\left[T_{\text {round }}\right]} \cdot t_{\text {data }} \\
& =\frac{\frac{N}{K^{N}} \sum_{k=1}^{K}(k-1)^{N-1} \cdot t_{\text {data }}}{t_{\text {data }}+t_{0}+\frac{8}{250 K^{N}} \sum_{k=1}^{K} k\left(\frac{k}{K}\right)^{N}\left[1-\left(\frac{k-1}{k}\right)^{N}\right]} .
\end{aligned}
$$

Another relevant performance metric is the average delay for a successful transmission, which can be defined as $E[D]=$ $\frac{E\left[T_{\text {round }}\right]}{P_{N, K}}$. Notice that $E\left[T_{\text {round }}\right]$ represents the average round duration for both successful and collided transmissions, since a DATA packet is sent after DECISION period regardless of the number of winning contenders. This mechanism, however, is efficient as long as the length of the DATA packet is comparable with the average REQUEST length. When DATA packets are longer, it may be convenient to repeat the REQUEST phase until one transmitter has been cleared. We will come back later to how to design such a mechanism.

\section{A. Model validation}

We validate the analytical model with Monte Carlo simulations. To simplify the exposition of the results for different values of the Strawman resolution $K$ and number of contenders $N$, we define the ratio $\rho \triangleq K / N$. Figures 4(a)-4(c) show the success probability $P_{N, K}$ of a Strawman round, the average length $E\left[t_{r}\right]$ of a REQUEST phase, and the goodput $g$ versus the ratio $\rho$ for various number of contenders $N$. For each case, the analytical model nicely matches the numerical simulations.

Although the model equations (1)-(7) do not allow to determine an explicit solution to optimization problems that aim, for instance, to optimize $K$ in order to maximize the success probability or the network goodput for a given number of contenders $N$, they offer valuable insight to properly tune the Strawman mechanism in [7] and enhance its performance. Particularly, Figures 4(a)-4(c) show that the success probability $P_{N, K}$ of each round increases for increasing ratio $\rho$, irrespectively of the number of contenders $N$. A large $\rho$, however, may correspond to a large resolution $K=\rho N$, which can induce undesirable long REQUEST phase, eventually reducing the achievable goodput. To find a good tradeoff, Table I presents the ratio $\rho^{\star}$ that maximizes the network goodput for the number of contenders $N$ used in Figure 4(c). For small $N$, the success probability of each round $P_{N, K}$ can be enhanced without significantly affecting $E\left[t_{r}\right]$ by choosing the ratio $\rho^{\star}$ in the range $[5,7]$. For a large number of contenders $N$, however, the optimal goodput occurs at smaller $\rho$ which keeps the resolution $K$, and hence $E\left[t_{r}\right]$, to reasonable values. 
TABLE I

THE RESOLUTION $K^{\star}$ WHICH MAXIMIZES GOODPUT IN FIGURE 4(C) FOR DIFFERENT NUMBER OF CONTENDERS $N$.

\begin{tabular}{ccccccc}
\hline Number of contenders $N$ & 5 & 10 & 25 & 50 & 75 & 100 \\
\hline$\rho^{\star}=K^{\star} / N$ & 7 & 5 & 3.25 & 2.25 & 2 & 1.75 \\
\hline
\end{tabular}

\section{B. Parameter tuning with uniform distribution}

We next exploit the insight offered by Figures 4(a)-4(c) to modify the baseline Strawman mechanism [7] while keeping the uniform distribution. The objective is to increase the success probability at each round, while keeping the average length of the REQUEST packets as short as possible.

The new mechanism runs in two steps: the first step is a Strawman round where the parameters are initialized using the guidelines from Table I. If a new collision occurs, a self-tuning step is triggered to maximize the probability of success in all subsequent rounds while reducing the length of the REQUEST phase. For this end, we let only the colliding transmitters participate in this step until a successful transmission occurs. Although the number of colliding nodes is unknown to both senders and receiver, the surviving transmitters can re-tune the Strawman resolution $K$ based on the estimated average of colliding nodes $E[\tilde{N}]$. Specifically, Figure 5, compares the average number of winners $E[\tilde{N}]$ from (5) against simulations for a fixed resolution $K=N$, showing that this number stabilizes around 1.55. Removing the bias induced by the successful rounds (i.e. $\tilde{N}>1$ ), we observe that the average number of colliding transmitters is approximately $E[\tilde{N}] \approx 2.5$ for all $N$, with relatively small standard deviation. Exploiting this result, we re-tune the resolution as $\tilde{K}=\tilde{\rho} E[\tilde{N}]$, where one can use $E[\tilde{N}] \approx[2,3]$ and choose $\tilde{\rho} \in[2,7]$ to guarantee a high success probability. Hence, the new resolution $\tilde{K}$ is a design parameter known at each node from the beginning. Upon the first collision, the colliding nodes change resolution from $K$ to $\tilde{K}$ and compete again with a new Strawman round as summarized in Algorithm 1.

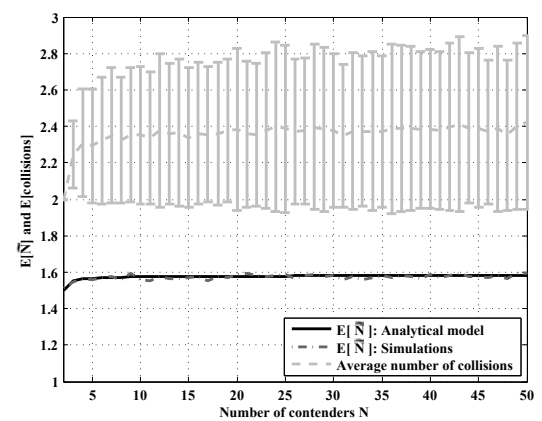

Fig. 5. Average number of winning contenders $E[\tilde{N}]$ and standard deviation in each round for $\rho=1$ and $N \in[1,50]$.

\section{STRAWMAN OPTIMIZATION}

Although the analytical model proposed in Section IV allows to optimally tune the parameters of the basic Strawman mechanism, the use of a uniform probability distribution to draw the request length remains, in general, suboptimal. In

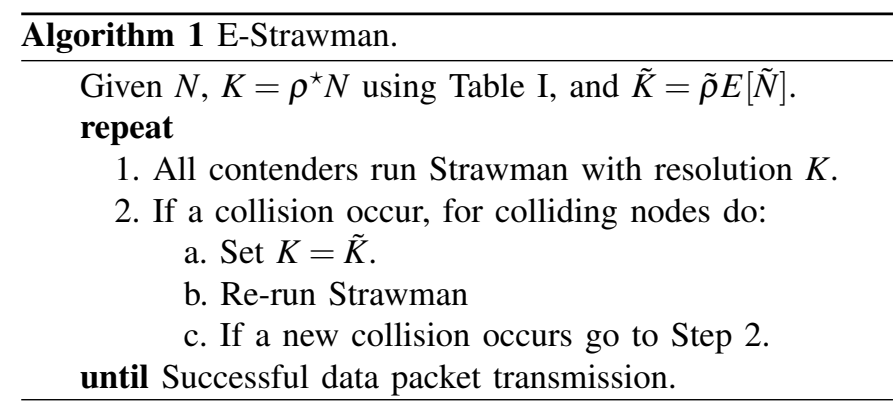

what follows we derive the optimal distribution for the Strawman mechanism in a sense that maximizes the probability of success $P_{N, K}$ for given $N$ and $K$. Our analysis in many aspects is similar to the problem studied by Tay et al. [10] to minimize the collision probability of CSMA-based protocols.

\section{A. Optimal probability distribution}

Given $N \geq 2$ contenders and contention window $K$, Tay et al. [10] derived the optimal distribution for CSMA-based protocols by defining the following recursive function $f_{k}(N)$.

Definition 5.1: Given a (slot) number $k \in[1, K]$ and $N \geq 2$ contenders let $f_{k}(N)$ be defined as

$$
f_{1}(N)=0, \text { and } f_{k}(N)=\left(\frac{N-1}{N-f_{k-1}(N)}\right)^{N-1} \forall k \geq 2 .
$$

One can show by induction that

$$
f_{k-1}(N)<f_{k}(N)<1 \quad \forall k \geq 2 .
$$

Although originally thought for sender-initiated protocols, we next prove that $f_{k}(N)$ can be used for deriving the optimal request length distribution $p^{\star}$ for Strawman.

Consider the Strawman contention resolution mechanism with given number of contenders $N$ and resolution $K$. Each contender randomly picks a number $k \in[1 \ldots K]$ independently with probability $p_{k}$. Let $p$ be the associated probability mass function. The success probability $P_{N, K}$ when $N$ contenders draw straws with this probability mass function is

$$
\begin{aligned}
P_{N, K}= & N p_{K}\left(1-p_{K}\right)^{N-1}+N p_{K-1}\left(1-p_{K}-p_{K-1}\right)^{N-1} \\
& +\cdots+N p_{1}\left(1-p_{K}-p_{K-1}-\cdots-p_{1}\right)^{N-1} \\
= & N \sum_{k=1}^{K} p_{k}\left(1-\sum_{r=k}^{K} p_{r}\right)^{N-1} .
\end{aligned}
$$

The following lemma provides an expression for the first-order optimality conditions for $p$ to maximize $P_{N, K}$ :

Lemma 5.2: Given a probability distribution $p$, if $\frac{\partial}{\partial p_{j}}\left(\frac{p_{N, K}}{N}\right)=0$ for $j=2, \ldots, K$, then

$$
\left(N-f_{j-1}(N)\right) p_{j}=\left(1-f_{j-1}(N)\right)\left(1-\sum_{r=j+1}^{K} p_{r}\right) .
$$

Proof: The result follows from verifying $\frac{\partial}{\partial p_{j}}\left(\frac{p_{N, K}}{N}\right)=$ 0 and induction. Due to space limitation, we omit the proof details. The interested reader may refer to [22].

The following theorem defines the optimal probability distribution for Strawman. 
Theorem 5.3: Given $N \geq 2$ contenders and a resolution $K$, the probability distribution $p^{\star}$ that maximizes Strawman success probability $P_{N, K}$ over all distributions $p$ is

$$
p_{k}^{\star}=\frac{1-f_{k-1}(N)}{N-f_{k-1}(N)}\left(1-\sum_{r=k+1}^{K} p_{r}\right) \forall k=2, \ldots, K,
$$

Proof: Essentially, one can show that the maximum $P_{N, K}$ occurs at an interior point of the interval $[0,1]$ for all $k$. Moreover, since $p_{1}=1-\sum_{k=2}^{K} p_{k}$ the $\max _{p_{2}, \ldots, p_{K}} P_{N, K}$ must necessarily occur where $\frac{\partial}{\partial p_{j}}\left(\frac{p_{N, K}}{N}\right)=0$ for all $j=2, \ldots, K$, for which Lemma 5.2 identifies $p^{\star}$ in (12) as the unique solution. Inspection of the second derivatives verifies that this solution indeed yields a maximum.

Lemma 5.4: With $N=2$ contenders and resolution $K$, the optimal probability distribution $p^{\star}$ is a uniform distribution.

Proof: For $N=2$, it follows from (12) that $p_{2}^{\star}=$ $\frac{1}{N}\left(1-\sum_{r=3}^{K} p_{r}\right)=\frac{1}{N}\left(p_{1}^{\star}+p_{2}^{\star}\right)$, hence $p_{2}^{\star}=\frac{1}{N-1} p_{1}^{\star}$. Also for $k=3$ (12) results in $p_{3}^{\star}=\frac{N}{(N-1)^{2}} \frac{p_{1}^{\star} p_{2}^{\star}}{p_{1}^{\star}+p_{2}^{\star}}$ and consequently, $p_{3}^{\star}=$ $\frac{1}{(N-1)^{2}} p_{1}^{\star}$. Similarly, it follows by recursive computation that $p_{k}^{\star}=\frac{1}{(N-1)^{k-1}} p_{1}^{\star}$. Summing the probabilities eventually leads to $p_{1}^{\star}+\frac{p_{1}^{\star}}{N-1}+\cdots+\frac{p_{1}^{\star}}{(N-1)^{K-1}}=1$. Hence, with $N=2$ contenders $p_{k}^{\star}=\frac{1}{K} \forall k$, i.e. a uniform distribution is optimal.

For the general case of $N>2$ contenders, selecting a uniform distribution as in the original Strawman design in [7] is suboptimal. The optimal probability distribution $p^{\star}$ can be computed numerically by first computing $f_{k}(N)$ for all $k=1, \ldots, K$ using (8), and then applying (12) to compute $p_{k}^{\star}$ recursively from $k=K$ backwards. The recursive computation of $f_{k}(N)$ with Eq. (8) takes $O(K)$ arithmetic operations, while the backward loop (12) takes $O(K)$ steps as well. Therefore, the computational complexity of the optimal probability distribution is linear in the resolution $K$.

\section{B. Approximating the optimal distribution}

Although computing the optimal probability is not too expensive, it does not provide a closed form probability distribution function. To grasp a better understanding of the optimal distribution, in this section we study two different approximation methods that simplify the computation of the optimal distribution, but nonetheless, have a success probability close to optimal. The advantage of such approximations is that with small modifications one can re-use them for other scenarios (e.g., adapted for CSMA-like protocols).

Figure 6 shows the optimal request length probability mass function for $N=3$ and $N=8$ contenders, respectively, with resolution $K=8$, along with the region where the optimal distribution $p^{\star}$ takes values starting from $N=3$ to $N=\infty$. First we observe that the shape of the optimal distribution $p^{\star}$ resembles a geometric distribution. Starting from relatively high $p_{1}^{\star}$, the tail of the distribution decays when we move to the larger numbers.

1) SIFT approximation: We first propose to use a geometric approximation similar to SIFT [10].

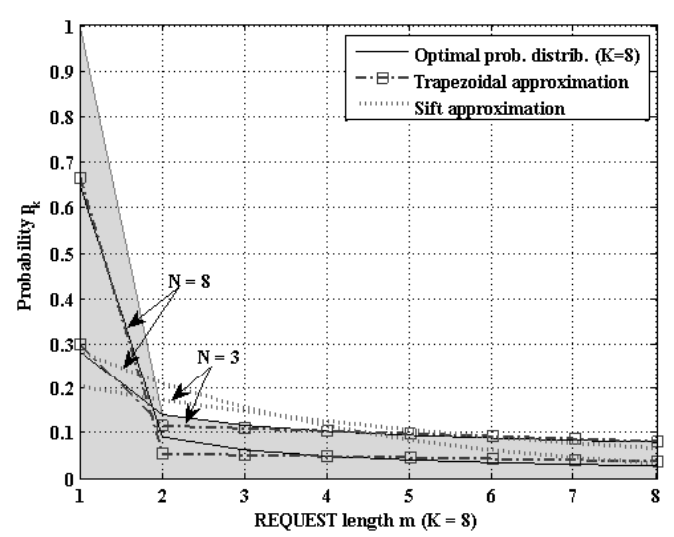

Fig. 6. Optimal probability distribution $p^{\star}$ and two approximation methods for $N=3,8$ contenders and $K=8$. The shaded area represents the region where $p^{\star}$ moves for $N \geq 3$. Starting from $N=3$, the optimal mass distribution for $k \in[2, K]$ decreases with increasing $N$ and it is redistributed in $p_{1}^{\star}$. The truncated geometric distribution Sift tends to overestimate $p^{\star}$ for $k \in[2, K]$ and underestimate $p_{1}^{\star}$, while the trapezoidal approximation offers a better fit.
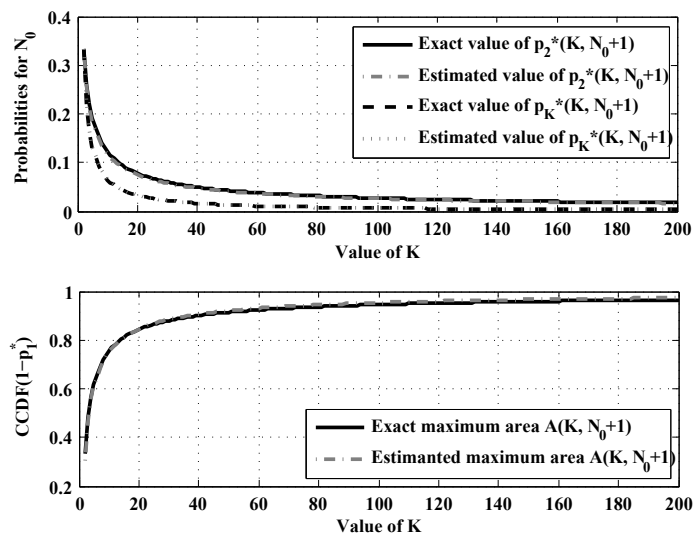

Fig. 7. Comparison of exact and approximated values of $p_{2}^{\star}, p_{K}^{\star}$, and $\hat{A}$ for the case $\left(K, N_{0}+1\right)$ using equations (15), (16) and (17), respectively.

Result 5.5: Given $N$ contenders and resolution $K$, the optimal request length probability distribution $p^{\star}$ can be approximated with a truncated geometric distribution of the form

$$
p_{k}=\frac{p \cdot q^{k-1}}{1-q^{K}},
$$

where $p=1-N^{\frac{-1}{K-1}}$ and $q=1-p$.

Due to space limitations, we omit the details that lead us to this result; The interested reader may refer to [22].

Now, similarly to Section IV, we can analyze Strawman using the approximate probability distribution in (13). Specifically, the success probability can be rewritten as

$$
P_{N, K}=\frac{N}{1-q^{K}} \sum_{k=1}^{K} p \cdot q^{k-1}\left(1-q^{k-1}\right)^{N-1} .
$$

By repeating the steps of Eq. (3)-(7), one can compute the average length of a request round (in bytes) $E[x]$, REQUEST phase duration $E\left[t_{r}\right]$, and goodput respectively.

2) A trapezoidal approximation: Although the previous approximation distributes probabilities coherently with the 
shape of the optimal probability distribution $p^{\star}$, Figure 6 shows that this approximation is not very tight. Particularly, for relatively high $p_{1}^{\star}$ the mass of the geometric distribution tends to be concentrated around the first small values of $k$, yielding a high probability of collision. The optimal distribution $p^{\star}$, on the other hand, assigns a larger probability to $k=1$ and has a fatter (and flatter) tail than a geometric distribution, thus allowing larger straws $k$ to be drawn, yielding lower collision probability. In what follows we propose an alternative approximation that aims at imitating the shape of the optimal distribution more closely. The approximation is inspired by the shape of the distribution for $k \geq 2$.

Let $p^{\star}(K, N)$ denote the optimal probability distribution for a given $K$ and $N$, and consider $\left(K_{0}, N_{0}\right)=(3,2)$ for which the optimal probability mass function is $p_{k}^{\star}\left(K_{0}, N_{0}\right)=\frac{1}{3}$ for $k=$ $1, \ldots, 3$. We now proceed numerically: Figure 7 suggests the following relation between $p_{2}^{\star}\left(K_{0}, N_{0}\right)$ and $p_{2}^{\star}\left(K, N_{0}+1\right)$

$$
\hat{p}_{2}^{\star}\left(K, N_{0}+1\right)=p_{2}^{\star}\left(K_{0}, N_{0}\right)\left(\frac{K_{0}}{K}\right)^{0.65} \quad K \geq K_{0},
$$

while $p_{K}^{\star}\left(K_{0}, N_{0}\right)$ and $p_{K}^{\star}\left(k, N_{0}+1\right)$ are related via

$$
\hat{p}_{K}^{\star}\left(K, N_{0}+1\right)=p_{K}^{\star}\left(K_{0}, N_{0}\right)\left(\frac{K_{0}}{K}\right) \quad K \geq K_{0} .
$$

Furthermore, the complementary cumulative distribution function $(\mathrm{CCDF})\left(A \triangleq 1-p_{1}^{\star}=\sum_{k=2}^{K} p_{k}^{\star}\right)$ is approximately

$$
\hat{A}\left(K, N_{0}+1\right)=1-\frac{\log \left(K+K_{0}\right)}{K} \quad K \geq K_{0} .
$$

Finally, we draw similar plots for the CCDF for the optimal distribution and $N>N_{0}$. These plots reveal the following area approximation $\hat{A}(K, N)$ for arbitrary $K>K_{0}, N>N_{0}$ :

$$
\hat{A}(K, N)=\hat{A}\left(K, N_{0}+1\right)\left(\frac{N_{0}+1}{N}\right)^{\frac{3}{4}} \text {. }
$$

Figure 8 shows the accuracy of the approximation in (18) for resolution $K=8,32$ and up to $N=200$ contenders.

Our second step is to make a linear interpolation of request length probabilities between $p_{2}^{\star}$ and $p_{K}^{\star}$ and assigning the remaining probability mass to $p_{1}$. Let $\theta(K)=\frac{\hat{p}_{2}^{\star}\left(K, N_{0}+1\right)}{\hat{p}_{K}^{\star}\left(K, N_{0}+1\right)}$ denote the estimated ratio between the extreme points of $p^{\star}$ in the region $[2, K]$ for $N=N_{0}+1$. By approximating the optimal probability distribution $p^{\star}$ between $[2, K]$ with a trapezoidal shape, we can estimate the values of $p_{2}^{\star}$ and $p_{K}^{\star}$ as

$$
\begin{array}{ll}
\hat{p}_{2}^{\star}(K, N)=\frac{2 \theta(K)}{1+\theta(K)} \frac{\hat{A}(K, N)}{K-1} & \forall K>K_{0}, N>N_{0} \\
\hat{p}_{K}^{\star}(K, N)=\frac{2}{1+\theta(K)} \frac{\hat{A}(K, N)}{K-1} & \forall K>K_{0}, N>N_{0} .
\end{array}
$$

Finally, the estimates $\hat{p}_{k}^{\star}(K, N)$ with $k=3, \ldots K-1$ can be obtained by a simple linear interpolation between $\hat{p}_{2}^{\star}(K, N)$ and $\hat{p}_{K}^{\star}(K, N)$, while $\hat{p}_{1}^{\star}(K, N)=1-\sum_{k=2}^{K} \hat{p}_{k}^{\star}(K, N)$. Taking a closer look at Figure 6, one can compare the trapezoidal and the geometric approximations for $K=8$ and $N=3,8$. While the truncated geometric distribution tends to overestimate $p^{\star}$ for $k \in[2, K]$ and largely underestimates $p_{1}^{\star}$, the trapezoidal approximation offers a better fit.
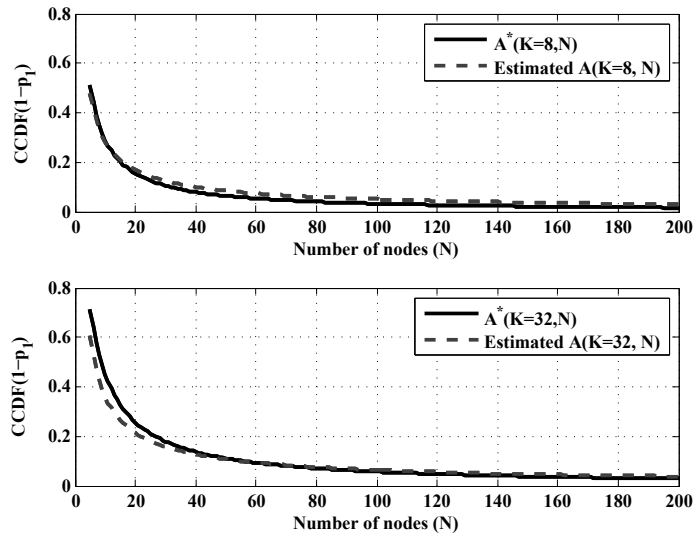

Fig. 8. Comparison of the CCDF of optimal distribution and the estimated area in equation (18) for resolution $K=8,32$ and $N \in[2,200]$ contenders.

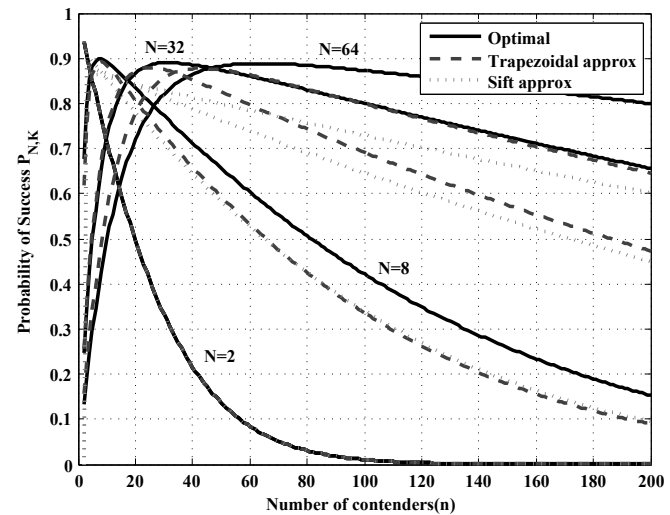

Fig. 10. Comparison of success probability tuned for $N=\{2,8,32,64\}$ and resolution $K=16$. The actual number of contenders ranges in $n \in[1,200]$. The maximum $P_{N, K}$ happens when $n=N$.

\section{Evaluation}

We evaluate how our improved Strawman mechanism performs in goodput, reliability, and scalability, and compare it against previously proposed Strawman mechanism [7]. We also compare the performance of receiver initiated contention resolution with sender initiated random backoff.

With the hidden terminal profile from Section III, we can perform experiments in controlled environments, to study the impact of both hidden terminals and the capture effect. Note that although Strawman would too benefit from capture effect, we choose to not enable it in the Strawman experiments.

We simulate two different Strawman implementation overheads: ideal and realistic. We base the ideal overhead on the CC2420 radio datasheet [12]: $t_{p r}=0.192 \mu \mathrm{s}$ and $t_{r d}=0.300 \mu \mathrm{s}$. The realistic overhead, in contrast, is obtained from measurements on our implementation on Contiki and the TmoteSky sensor platform: $t_{p r}=1.1 \mathrm{~ms}$ and $t_{r d}=1.2 \mathrm{~ms}$. CSMA is evaluated only with an idealistic overhead, as we have no corresponding implementation available.

\section{A. Optimizing Uniform Strawman}

We first compare the performance of the basic Strawman mechanism [7] with our enhanced version, E-Strawman, proposed in Section IV for REQUEST lengths drawn using a 


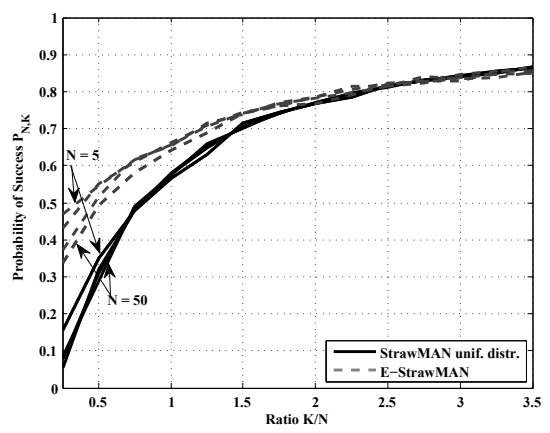

(a) Round success probability

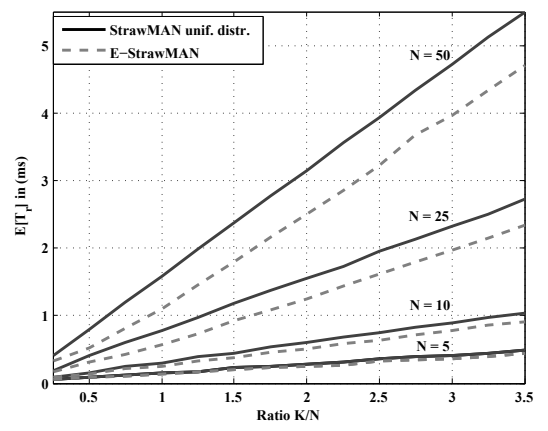

(b) Average request length.

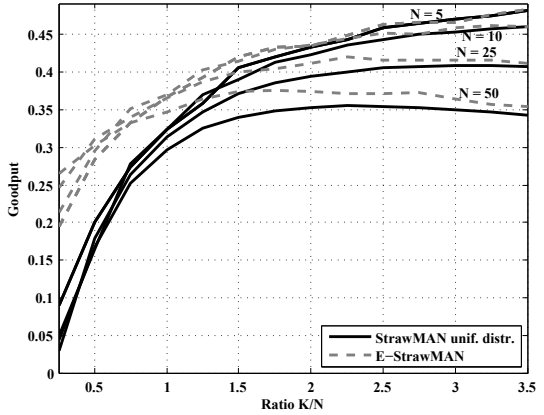

(c) Goodput.

Fig. 9. Comparison of StrawMAN and E-StrawMAN for uniform probability distribution. We plot the success probability $P_{N, K}$ of a round, the average request length $E\left[t_{r}\right]$ and the goodput $g$ against the ratio $\rho=K / N$ for $N=\{5,10,25,50\}$ contenders.

uniform probability distribution. Figure 9 compares the success probability, average request length and goodput of Strawman and E-Strawman. Figure 9(b) shows that E-Strawman strongly reduces the average request length. Not surprisingly, E-Strawman offers higher success probability and goodput for ratios $\rho<2$, while it converges to the same performance of Strawman for higher values of $\rho$. Essentially, the performance gain of E-Strawman vanishes for high values of $\rho$ where Strawman exhibits a fairly high success round probability. However, for a large number of contenders $N$, Strawman is optimal to work with small values of $\rho$ where E-Strawman typically outperforms Strawman.

\section{B. Robustness of the optimal distribution and approximation}

As proven in Section $\mathrm{V}$, drawing the request lengths $x_{n}$ from a uniform distribution is optimal only for $N=2$ contenders. On the other hand, given a resolution $K$, the probability distribution $p^{\star}$ derived in Theorem 5.3 is optimal only if the number of contenders is exactly $N$. The effective number of contenders $n$, however, is typically unknown and needs to be estimated. In what follows we will refer to $N$ as the estimated number of contenders, and we evaluate the robustness of the optimal distribution, as well as the trapezoidal and SIFT approximations, when $N \neq n$.

To this end, Figure 10 shows the success probability of a Strawman round with three distributions computed for $N=$ $2,8,32,64$, when the effective number of contenders ranges in $n \in[2,200]$. Not surprisingly, the maximum value of the success probability using $p^{\star}$ occurs at $n=N$. More interestingly, we notice that overestimating the number of contenders $N$, i.e. for $N>n$, can potentially lead to small success probability using either of the distributions. Essentially, if $N$ is larger than the effective number of contenders, the distribution $p^{\star}$ and its approximations overestimate the optimal $p_{1}$, thus letting most of the contenders draw small numbers and eventually producing multiple winners, hence a collision. To the contrary, underestimating the number of contenders (i.e. for $N<n$ ) is less harmful. In either case, the trapezoidal approximation always yields higher success probability than its counterpart SIFT. More importantly, Figure 10 shows that if $N$ is in a

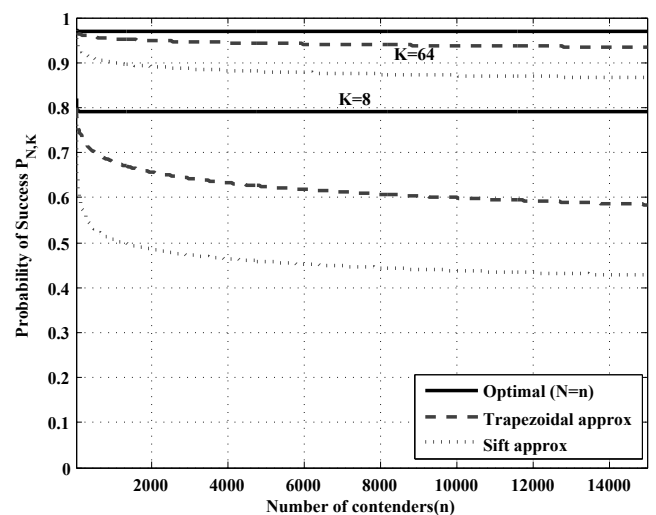

Fig. 11. Scalability of trapezoid approximation: the plot shows that with large number of contenders trapezoid approximation decays smoothers than sift approximation. the experiment is conducted with $K=8,64$.

range of $20 \%-30 \%$ from the effective number of contenders $n$, the performance loss is small compared to the case $n=N$.

Another important aspect is the scalability. To this end, Figure 11 illustrates the success probability of a Strawman round with optimal distribution, trapezoidal and SIFT approximations, respectively, computed for two fixed resolutions $K=8$ and $K=64$ with $n=N$ for a large number of contenders ${ }^{1}$. We observe that the optimal success probability of a strawman round when the distribution $p^{\star}$ is tuned to the exact number of contenders only depends on the resolution $K$, with better performance for higher $K$. The solid line corresponds to the envelope of the maximum points of Figure 10 which happens when $n=N$. In all cases, the trapezoidal approximation always yields better performance than the SIFT approximation. Combined with the insights from Figure 10, similar results can be obtained when the estimated $N$ in a range of $20 \%-30 \%$ from the effective number of contenders.

\section{Experimental validation}

We next validate the Strawman mechanism with trapezoidal approximation designed with resolution $K=16$ and $N$ ranging

\footnotetext{
${ }^{1}$ Obviously, such a high range of contenders is not intended to reflect any practical scenario, but only to analyze numerically the protocol behavior.
} 

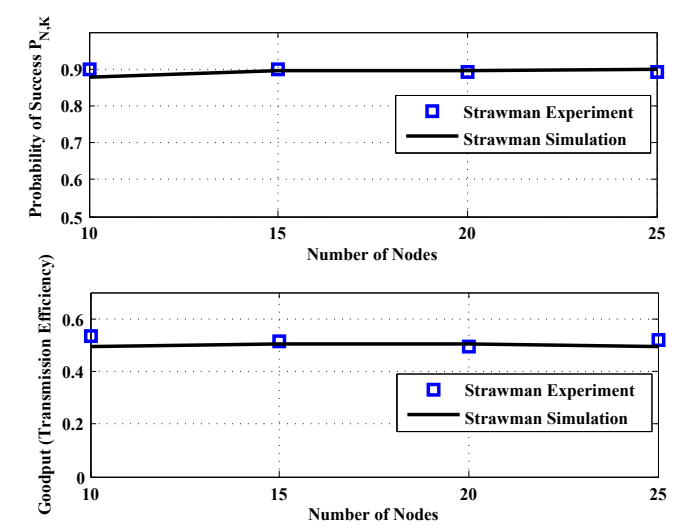

Fig. 12. Success probability and goodput validation with experimental results for Strawman mechanism using the trapezoidal approximation design with $K=16$ and $N=10,15,20$, and 25 nodes.

from 10 to 25 contenders. Figure 12 shows a very accurate match between the simulation and experimental values of the success probability and goodput of Strawman for all cases. Leveraging on this match, we will continue our evaluation through extensive simulations.

\section{Idealistic Networks: No Hidden Terminal Problem}

In the first experiment we simulate Strawman in a network without any hidden terminals, and measure reliability and goodput. A large number of (interfering) collisions lowers both the reliability and the goodput metrics, whereas a large protocol overhead majorly affects the goodput metric.

Figure 13(a) compares the reliability of Strawman with CSMA contention resolution mechanism. Our first observation regarding to the optimal and approximation distributions of Strawman is that the performance of Strawman in these cases remains mostly unchanged with respect to increasing number of contenders. This behavior is due to the scalability property of these distributions and confirms the results of Figure 11.

Figure 13(a) shows that CSMA and Strawman with a uniform distribution do not scale well with increasing number of contenders. For instance, considering Strawman with uniform distribution and using $\mathrm{K}=16$, the parameter $\rho=K / N$ decreases from $16 / 5$ to $16 / 100$. Now, we can observe two things. First, for $\rho=16 / 5 \approx 3$ Strawman and E-Strawman have roughly the same performance (which is confirmed by the same starting point in Figure 9 shows). Second, for decreasing $\rho$ (i.e., by increasing $N$ ), E-Strawman becomes better than the basic Strawman. However, the resolution used for each $N$ is smaller than the $K^{\star}$ recommended in Table I; since we are moving to smaller values of $\rho$ both methods introduce poor performance.

Figure 14(a) shows the experiment goodput. Note how the implementation overhead affects Strawman's goodput: the realistic overhead vs the ideal overhead.

\section{E. Hidden Terminals Without Capture Effect}

Strawman, in contrast to CSMA, is designed to cope with the hidden terminal problem. We now include hidden terminals as profiled in Section III. We do not, however, yet include the (positive) effects of the capture effect phenomenon. Neither
Strawman nor CSMA is designed to exploit the capture effect, and so it is interesting to study how they behave without capture effect. Moreover, capture effect efficiency differs with network types and radio hardware.

Figure 13(b) shows how the reliability of the mechanisms is affected by hidden terminals. Whereas Strawman is unaffected by the addition of hidden terminals, CSMA suffers significantly. The goodput experiments, as shown in Figure 14(b), show that CSMA networks deliver almost no data to the receiver due to interfering packet collisions.

\section{F. Testbed Profile: Hidden Terminals and Capture Effect}

We finally enable the capture effect phenomenon, thus fully mimicking the testbed in Section III. For CSMA, we can observe an increase in both reliability (Figure 13(c)) and goodput (Figure 14(c)). Strawman is not simulated with capture effect in these experiments, and thus has the same performance. As these experiments show, Strawman outperforms CSMA even when CSMA benefits from capture effect.

We observe an interesting phenomenon in these experiments: CSMA with uniform distribution appears to perform better with more contenders. At first glance, this is highly counter-intuitive: CSMA with uniform distribution was shown to scale badly even without hidden terminals (Figure 13(a) and Figure 14(a)). After careful studying of experiment logs we attribute this behavior to a complex interaction between capture effect and the random backoff-distribution. The uniform distribution renders more collisions at early stages of a transmission, whereas the SIFT distribution achieves a higher probability of a single transmission. If a neighbor in the uniform-based network manages to successfully initiate a transmission to the receiver (due to capture effect), the probability is high that several other neighbors are also transmitting (with a lower signal strength), thus blocking the rest of the network from interfering transmissions. This initial result motivates us to further study the relation between capture effect and hidden terminals, and demonstrates that protocols must be evaluated in realistic but controlled environments.

This evaluation has compared Strawman with CSMA in three scenarios with increasing realism. We observe that hidden terminals, with or without capture effect, may greatly degrade performance of contention resolution mechanisms. Strawman is, however, shown to yield the same high performance both with and without hidden terminals.

\section{CONCLUSIONS}

The Strawman contention resolution mechanism offers high performance and solves the hidden terminal problem. Using a hidden terminal testbed profile, we show that random backoff-based approaches suffer severe performance degradation whereas Strawman does not. We model the basic Strawman mechanism, and improve it with an optimal random length distribution. Our improved distribution outperforms the basic Strawman in both goodput and scalability. 


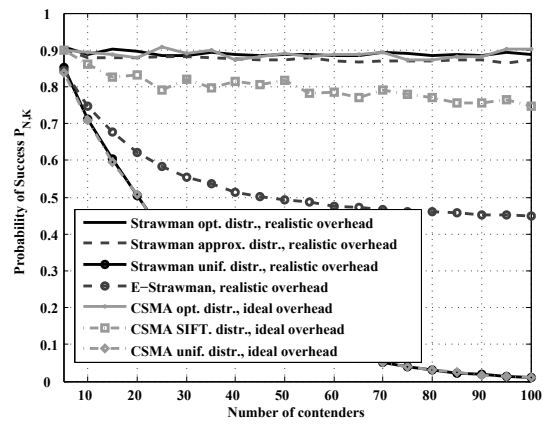

(a) Without hidden terminals

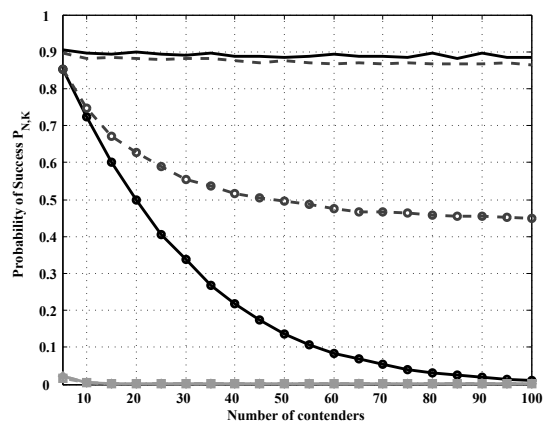

(b) Without capture effect

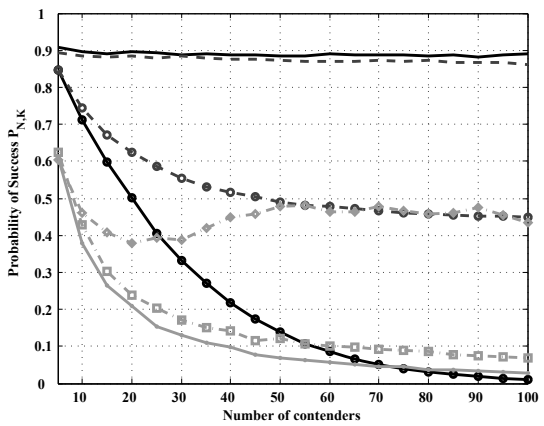

(c) Testbed profile

Fig. 13. The reliability - the ratio successful transmissions—of Strawman remains high in both (a) an idealistic network setting without hidden terminals, (b) with hidden terminals but without capture effect, and (c) with both hidden terminals and capture effect. The right-most network setting represents our testbed profile from Section III. Random backoff-based CSMA, in contrast, suffers from its inability to handle the hidden terminal problem. (All experiments assume a star network with $[5,100]$ senders and maximum length $K=16$.)

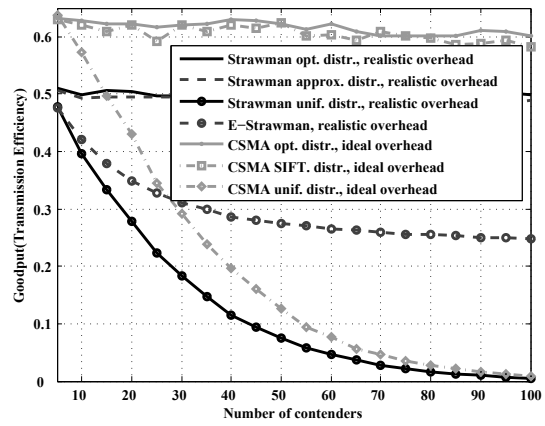

(a) Without hidden terminals

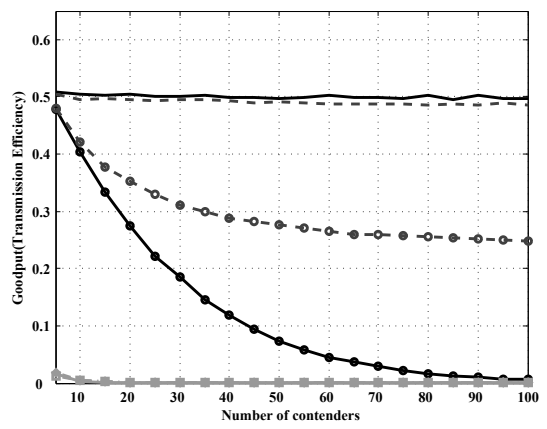

(b) Without capture effect

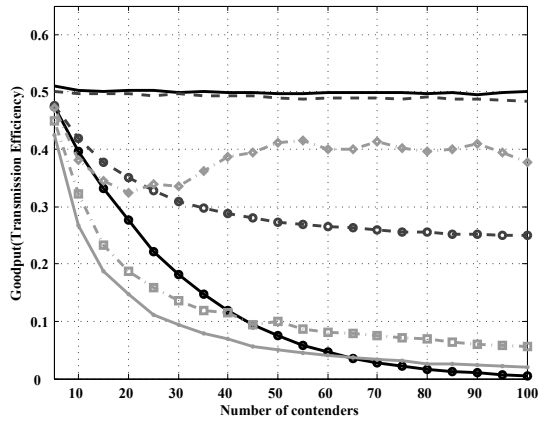

(c) Testbed profile

Fig. 14. The receiver goodput of Strawman is independent of the number of contenders. The Strawman ideal overhead graphs show the best achievable goodput, whereas the realistic overhead graphs show the performance based on our implementation overhead measurements. (a) As expected, SIFT outperforms Strawman in idealistic networks without hidden terminals. With the (b) addition of hidden terminals and (c) capture effect, however, Strawman has both significantly better scalability and higher goodput. (All experiments assume a star network with $[5,100]$ senders and maximum length $K=16$.)

\section{REFERENCES}

[1] O. Gnawali, R. Fonseca, K. Jamieson, D. Moss, and P. Levis. Collection tree protocol. In ACM SenSys, Berkeley, CA, USA, 2009.

[2] P. Levis, N. Patel, D. Culler, and S. Shenker. Trickle: A self-regulating algorithm for code propagation and maintenance in wireless sensor networks. In NSDI'04, March 2004.

[3] S. Kim, R. Fonseca, P. Dutta, A. Tavakoli, D. Culler, P. Levis, S. Shenker, and I. Stoica. Flush: A reliable bulk transport protocol for multihop wireless networks. In SenSys'07, Sydney, Australia, November 2007.

[4] A. Jindal and K. Psounis. Modeling spatially correlated data in sensor networks. ACM Transactions of Sensor Networks, 2007.

[5] Y. Sun, O. Gurewitz, and D. Johnson. RI-MAC: A Receiver-Initiated Asynchronous Duty Cycle MAC Protocol for Dynamic Traffic Loads in Wireless Sensor Networks. In ACM SenSys, Raleigh, NC, USA, 2008.

[6] P. Dutta, S. Dawson-Haggerty, Y. Chen, M. Liang, and A. Terzis. Design and Evaluation of a Versatile and Efficient Receiver-Initiated Link Layer for Low-Power Wireless. In ACM SenSys, 2010.

[7] F. Österlind, N. Wirström, N. Tsiftes, N. Finne, T. Voigt, and A. Dunkels. StrawMAN: Making sudden traffic surges graceful in low-power wireless networks. In ACM HotEMNETS, Killarney, Ireland, June 2010.

[8] M. Buettner, G. V. Yee, E. Anderson, and R. Han. X-MAC: A Short Preamble MAC Protocol for Duty-Cycled Wireless Sensor Networks. In ACM SenSys, pages 307-320, Boulder, Colorado, USA, 2006.

[9] J. Polastre, J. Hill, and D. Culler. Versatile low power media access for wireless sensor networks. In ACM SenSys, pages 95-107, 2004.

[10] Y. C. Tay, K. Jamieson, and H. Balakrishnan. Collision-Minimizing CSMA and its Applications to Wireless Sensor Networks. 2004.

[11] V. Handziski, A. Kpke, A. Willig, and A. Wolisz. TWIST: A Scalable and Reconfigurable Testbed for Wireless Indoor Experiments With Sensor Networks. In ACM REALMAN, 2006.

[12] Chipcon AS. CC2420 Datasheet (rev. 1.3), 2005.
[13] R. Musaloiu-E., C.-J. M. Liang, and A. Terzis. Koala. Ultra-low power data retrieval in wireless sensor networks.

[14] J.J. Garcia and A. Tzamaloukas. A receiver-initiated collision-avoidance protocol for multi-channel networks. In INFOCOM, 2001.

[15] F. Tobagi and L. Kleinrock. Packet Switching in Radio Channels: Part II. IEEE Trans. on Comm., December 1975.

[16] W. Ye, J. Heidemann, and D. Estrin. Medium Access Control with Coordinated, Adaptive Sleeping for Wireless Sensor Networks. In IEEE/ACM Trans. on Networking, Piscataway, NJ, USA, June 2004.

[17] A. El-Hoiydi, J.-D. Decotignie, C. C. Enz, and E. Le Roux. Wisemac, an ultra low power mac protocol for the wisenet wireless sensor network. In ACM SenSys, pages 302-303, 2003.

[18] A. Woo and D. Culler. A transmission control scheme for media access in sensor networks. In ACM MobiCom, 2001.

[19] K. Jamieson, H. Balakrishnan, and Y. Tay. Sift: A MAC protocol for event-driven wireless sensor networks. pages 260-275, February 2006.

[20] Kaixin Xu, Mario Gerla, and Sang Bae. Effectiveness of rts/cts handshake in ieee 802.11 based ad hoc networks. AdHocNet'03.

[21] J. Zhao and R. Govindan. Understanding packet delivery performance in dense wireless sensor networks. In ACM SenSys, 2003.

[22] E. Ghadimi, P. Soldati, F. Österlind, H. Zhang, and M. Johansson. Hidden Terminal-Aware Contention Resolution with an Optimal Distribution. Technical report, KTH, 2010. 